\title{
Medially based de-epithelialized flap for nasal base narrowing and nostril sill augmentation in a cleft lip nasal deformity
}

\author{
Kun Hwang', Jong Hwan $\mathrm{Choi}^{2}$, Hun Kim¹, Dae Joong Kim³ \\ ${ }^{1}$ Department of Plastic Surgery, Inha University School of Medicine, Incheon 400-711, South Korea. \\ ${ }^{2}$ Department of Plastic Surgery, Inha University Hospital, Incheon 400-711, South Korea. \\ ${ }^{3}$ Department of Anatomy, Inha University School of Medicine, Incheon 400-711, South Korea.
}

Correspondence to: Dr. Kun Hwang, Department of Plastic Surgery, Inha University School of Medicine, 27 Inhang-ro, Jung-gu, Incheon 400-711, South Korea. E-mail: jokerhg@inha.ac.kr

How to cite this article: Hwang K, Choi JH, Kim H, Kim DJ. Medially based de-epithelialized flap for nasal base narrowing and nostril sill augmentation in a cleft lip nasal deformity. Plast Aesthet Res 2016;3:291-5.

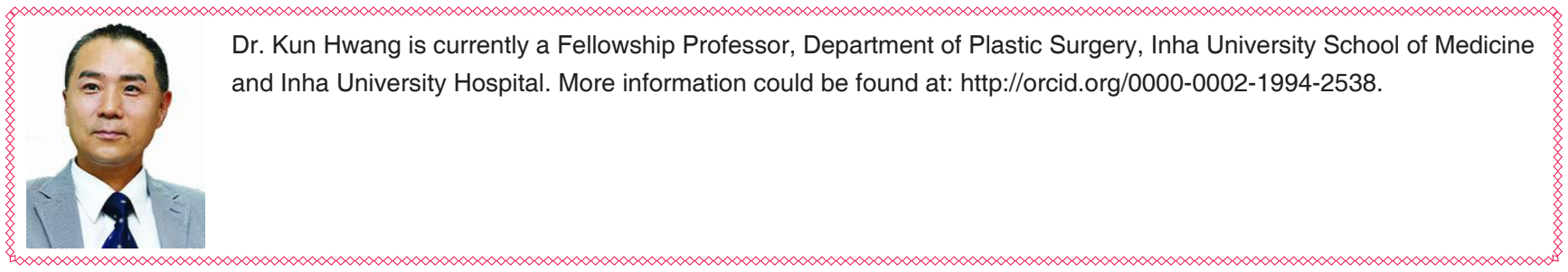

Article history:

Received: 22-08-2016

Accepted: 05-09-2016

Published: 20-09-2016

Key words:

Cleft lip,

nose deformities,

acquired,

surgical flaps,

dermis

\begin{abstract}
Aim: The authors observed the nostril floor in a gross cadaver specimen histologically and innovated a medially based de-epithelialized flap for nasal base narrowing and nostril sill augmentation. Methods: In cadaver, fully thick section was taken from the nostril sill at the midpoint of the columella base and ala base, and stained with Masson-Trichrome. In eight patients, circumferential incision along the nostril sill and alar base freed the alar base from the upper lip. At the columellar base, fresh epithelium was shaved on the medial side of the incision line. The widened scar on the upper lip was excised. The de-epithelialized tip of the columellar base was pulled under the medial tip of the alar base flap and sutured tightly. Four anthropometric distances were measured preoperatively and postoperatively. Results: Histologically the nostril sill was composed of thickened dermis. Just below the dermis, the depressor septi nasi muscle ran obliquely, augmenting the nostril sill. The nostril floor width, alar distance, and alar curvature distance decreased on the cleft side after the operation. Conclusion: A medially based de-epithelialized flap narrows the alar base and augments the nostril sill simultaneously, since the de-epithelialized part of the excess skin augments the depressed nostril sill.
\end{abstract}




\section{INTRODUCTION}

In patients with cleft lip nasal deformities, the upper lip scar is widened and the nasal base is wider than the unaffected side. Alar base reduction is an important technique for narrowing the frontal view of the nose. ${ }^{[1]}$

Moreover, the nostril sill is deficient on the affected side. Excision of the scar of the upper lip and nostril sill may leave a depressed nostril. ${ }^{[2]}$ Some authors have used laterally based alar flaps, ${ }^{[3]}$ stating that they were able to reduce the risk of notching by adapting a two-layer closure of the vestibular floor. ${ }^{[2]}$

We hypothesized that a medially based de-epithelialized flap might avoid the notching of the nostril sill, since the de-epithelialized part of the excess skin could augment
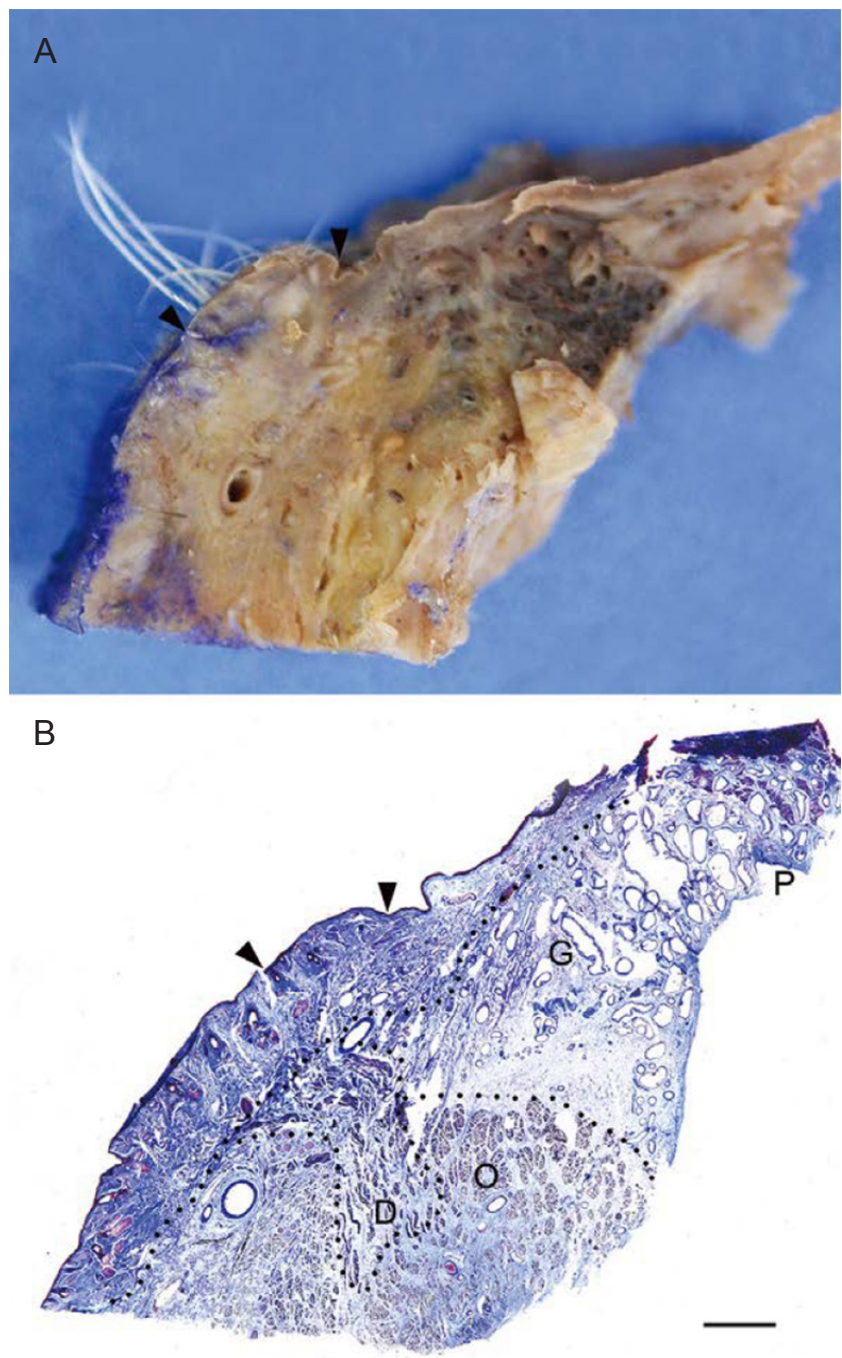

Figure 1: A perpendicular section of the nostril sill. Two arrowheads indicate the span of the nostril sill. A: Gross specimen; B: Histology with Masson trichrome stain, bar indicates $1.5 \mathrm{~mm}$. Note the thick dermis and the obliquely running depressor septi nasi muscle (D) comprising the nostril sill. O: orbicularis oris muscle; $\mathrm{P}$ : periosteum; G: nasal glands the depressed nostril sill.

We performed a histological observation of the nostril floor in a gross cadaveric specimen and created a medially based de-epithelialized flap for nasal base narrowing and nostril sill augmentation in cleft lip nasal deformities.

\section{METHODS}

\section{Cadaveric study}

On a cadaver, the nose including the upper lip was removed and fixed in $4 \%$ natural buffered formaldehyde. A fully thick section was taken from the nostril sill at the midpoint of the columellar base and the alar base. Following routine histologic procedures, the specimens were embedded in paraffin, sectioned at $10 \mu \mathrm{m}$, and stained with Masson trichrome. The prepared slides were observed under a light microscope. In the perpendicular section, the nostril sill was composed of thickened dermis. Just below the dermis layer, the depressor septi nasi muscle ran obliquely and augmented the nostril sill. No alar cartilage was found [Figure 1].

\section{Surgical technique}

A circumferential incision along the nostril sill and alar base freed the alar base from the upper lip. At the
A

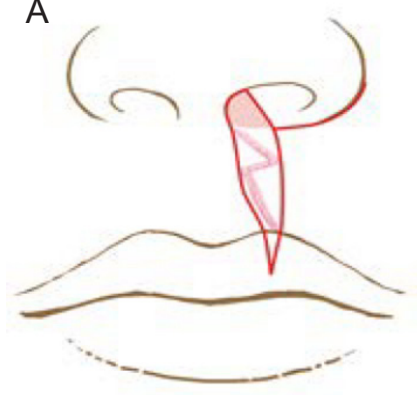

C

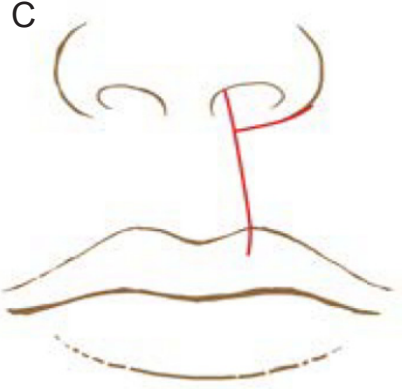

B

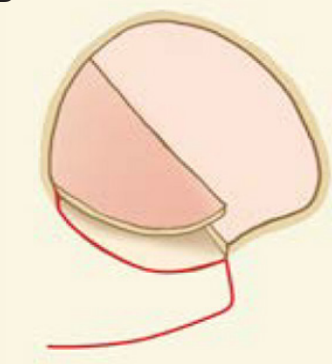

D

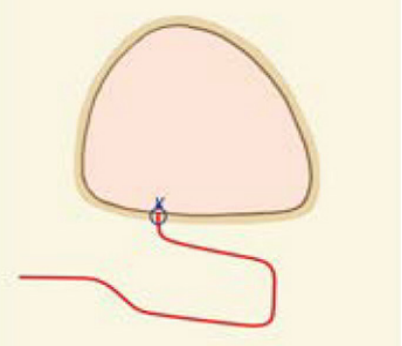

Figure 2: Surgical technique for the medially based de-epithelialized flap. (A and $B$ ): A circumferential incision along the nostril sill and alar base freed the alar base from the upper lip. At the columellar base, fresh epithelium was shaved on the medial side of the incision line. The widened scar on the upper lip was excised. (C and D): The raw (denuded and de-epithelialized) tip of the columellar base was pulled under the medial tip of the alar base flap and sutured tightly. (A and $C$ ): frontal view; (B and D): worm's eye view 


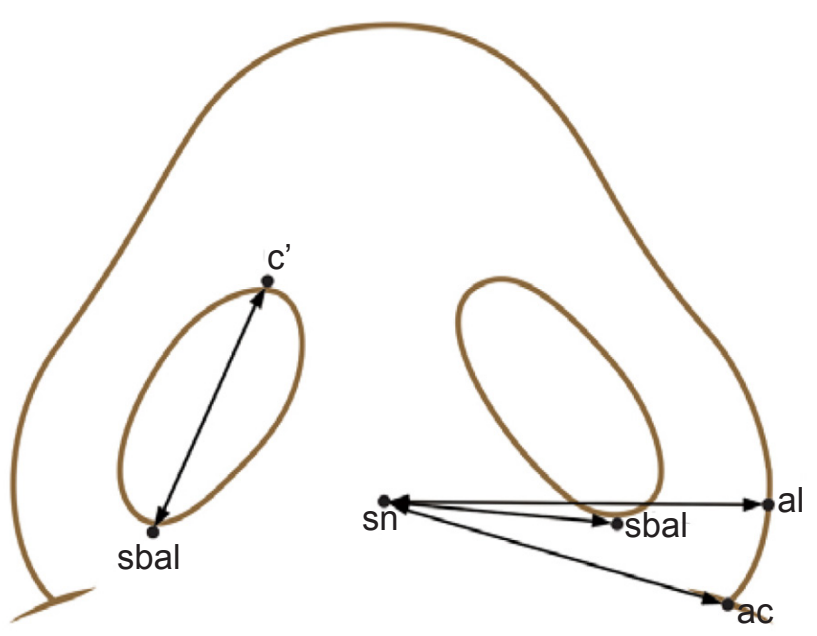

Figure 3: Four anthropometric distances were measured: nostril floor width (Sbal-Sn), alar distance (Sn-Al), alar curvature distance (Sn-Ac), and nostril length (Sbal-C'). al: alare; ac: alar curvature point; sn: subnasale; sbal: subalare; c': highest point of the columella

columellar base, fresh epithelium was shaved on the medial side of the incision line. The widened scar on the upper lip was excised. The raw (denuded and deepithelialized) tip of the columellar base was pulled under the medial tip of the alar base flap and sutured tightly. The nasal base was then narrowed and the nostril sill was augmented [Figure 2].

\section{Anthropometric measurements}

Four anthropometric distances were measured preoperatively and postoperatively using Adobe Photoshop CS2 version 9 (Adobe Systems Inc., San Jose, CA, USA). All values were obtained in pixels. In order to avoid personal bias and random systematic error, all measurements and statistical analyses were performed by a single researcher.

Four distances were measured on the cleft side and non-cleft side preoperatively and postoperatively [Figure 3]:[4,5] the nostril floor width (Sbal-Sn), the alar distance (Sn-Al), the alar curvature distance (Sn-Ac), and nostril length (Sbal-C'). These were transferred along the relative length to the intercanthal distance and the reduction rate $(R R)$ was calculated $[R R=$ (preoperative measurement - postoperative measurement)/preoperative measurement]. Statistical significance was evaluated using the independent two-sample $t$-test.

\section{Patients}

Nine patients ( 3 males and 6 females) were operated on using the medially based de-epithelialized flap technique. Among these 9 patients, 6 patients underwent followup for more than 12 months and their preoperative and postoperative worm's eye views were compared.

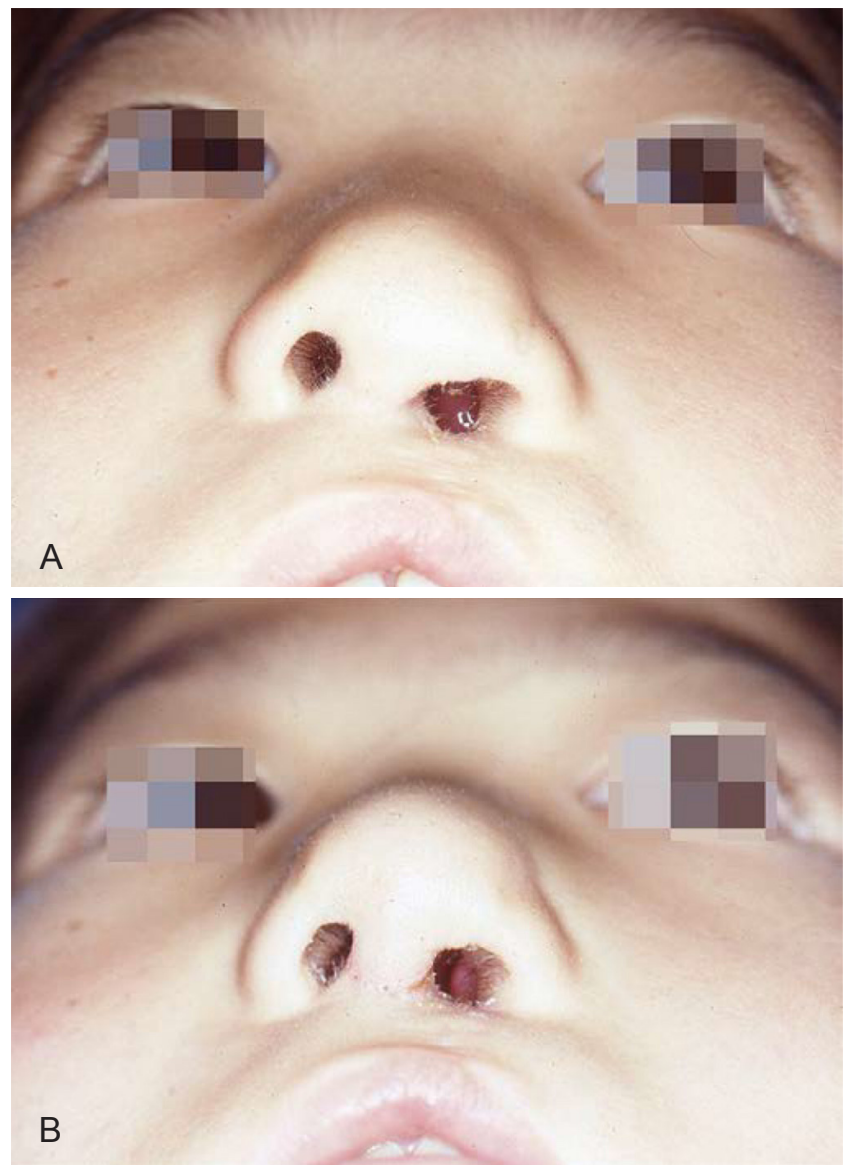

Figure 4: A 7-year-old girl with a cleft lip nasal deformity. The nostril sill was reduced using a medially based de-epithelialized flap. A: Preoperative worm's eye view; B: postoperative view

\section{RESULTS}

\section{Anthropometric results}

Although not to a statistically significant extent $(P>0.05$, independent two samples $t$-test) the nostril floor width (Sbal-Sn), alar distance (Sn-Al), and alar curvature distance (Sn-Ac) decreased on the cleft side after the operation. The RR of the nostril floor was $7.72 \% \pm$ $3.62 \%$. The RRs of the alar distance and alar curvature distance were $7.09 \% \pm 3.72 \%$ and $6.46 \% \pm 6.24 \%$, respectively [Table 1]. On the non-cleft side, the nostril floor width (Sbal-Sn), alar distance (Sn-Al), and alar curvature distance $(\mathrm{Sn}-\mathrm{Ac})$ did not change after the operation $(P>0.05$, independent two samples $t$-test). The RR of the nostril floor was $0.17 \% \pm 7.45 \%$. The RRs of the alar distance and alar curvature distance were $1.58 \% \pm 6.37 \%$ and $1.71 \% \pm 4.42 \%$, respectively [Table 1]. The nostril length (Sbal-C') increased on the non-cleft side $(6.17 \% \pm 15.60 \% ; P=0.04$, independent two samples $t$-test). The nostril length decreased on the cleft side $(2.95 \pm 30.81 \%)$, although this change was not statistically significant $(P>0.05$, independent two samples $t$-test) [Tables 1 and 2]. 
Table 1: Comparison of the reduction rate between the non-cleft and the cleft side (independent two samples $t$-test)

\begin{tabular}{|c|c|c|c|c|}
\hline \multirow{2}{*}{ Point } & \multirow{2}{*}{ Definition } & \multicolumn{2}{|c|}{ Reduction rate (\%) } & \multirow[b]{2}{*}{$P$-value } \\
\hline & & Non-cleft & Cleft & \\
\hline sbal-sn & Nostril floor width & $0.17 \pm 7.45$ & $7.72 \pm 3.62$ & 0.19 \\
\hline sn-al & Ala distance & $1.58 \pm 6.37$ & $7.09 \pm 3.72$ & 0.10 \\
\hline sn-ac & Ala curvature distance & $1.71 \pm 4.42$ & $6.46 \pm 6.24$ & 0.67 \\
\hline sbal-c' & Nostril length & $-6.17 \pm 15.60$ & $2.95 \pm 30.81$ & 0.25 \\
\hline
\end{tabular}

Data expressed in relative length of intercanthal distance. al: alare; ac: alar curvature point; c': highest point of the columella; sn: subnasale; sbal: subalare

Table 2: Comparison of non-cleft and cleft side preoperatively and postoperatively (independent $t$-test)

\begin{tabular}{|c|c|c|c|c|c|c|c|c|c|}
\hline \multirow{2}{*}{ Point } & \multirow{2}{*}{ Definition } & \multicolumn{3}{|c|}{ Non-cleft } & \multicolumn{5}{|c|}{ Cleft } \\
\hline & & Pre & & Post & $P$ & Pre & & Post & $P$ \\
\hline sbal-sn & Nostril floor width & $0.24 \pm 0.03$ & $=$ & $0.24 \pm 0.04$ & 0.47 & $0.29 \pm 0.06$ & $\geq$ & $0.27 \pm 0.04$ & 0.75 \\
\hline sn-al & Ala distance & $0.51 \pm 0.05$ & $=$ & $0.51 \pm 0.07$ & 0.22 & $0.61 \pm 0.06$ & $\geq$ & $0.57 \pm 0.06$ & 0.80 \\
\hline sn-ac & Ala curvature distance & $0.50 \pm 0.03$ & $=$ & $0.50 \pm 0.04$ & 0.35 & $0.62 \pm 0.05$ & $\geq$ & $0.58 \pm 0.04$ & 0.38 \\
\hline \multirow[t]{3}{*}{ sbal-c' } & Nostril length & $0.22 \pm 0.01$ & $<$ & $0.23 \pm 0.04$ & 0.04 & $0.21 \pm 0.04$ & $\geq$ & $0.20 \pm 0.05$ & 0.87 \\
\hline & & \multicolumn{3}{|c|}{ Pre-operation } & \multicolumn{5}{|c|}{ Post-operation } \\
\hline & & Non-cleft & & Cleft & $P$ & Non-cleft & & Cleft & $P$ \\
\hline sbal-sn & Nostril floor width & $0.24 \pm 0.03$ & $\leq$ & $0.29 \pm 0.06$ & 0.47 & $0.24 \pm 0.04$ & $\leq$ & $0.27 \pm 0.04$ & 0.91 \\
\hline sn-al & Ala distance & $0.51 \pm 0.05$ & $\leq$ & $0.61 \pm 0.06$ & 0.39 & $0.51 \pm 0.07$ & $\leq$ & $0.57 \pm 0.06$ & 0.59 \\
\hline sn-ac & Ala curvature distance & $0.50 \pm 0.03$ & $\leq$ & $0.62 \pm 0.06$ & 0.12 & $0.50 \pm 0.04$ & $\leq$ & $0.58 \pm 0.04$ & 0.79 \\
\hline sbal-c' & Nostril length & $0.22 \pm 0.01$ & $\geq$ & $0.21 \pm 0.04$ & 0.10 & $0.23 \pm 0.04$ & $\geq$ & $0.20 \pm 0.05$ & 0.85 \\
\hline
\end{tabular}

Data expressed in relative length of intercanthal distance. al: alare; ac: alar curvature point; c': highest point of the columella; sn: subnasale; sbal: subalare. $=$ : same mean value; $\geq$ and $\leq$ : no significant difference $(P>0.05)$; <: significant difference $(P<0.05)$

\section{Patient case}

A 7-year-old girl had a cleft lip nasal deformity. Her nasal tip was augmented with a conchal cartilage graft and her nostril sill was reduced with a medially based deepithelialized flap [Figure 4].

\section{DISCUSSION}

A nasal base reduction typically involves the soft tissue resection of one or more of the nasal bases (ala, sill, or columellar base).[6] Anatomically, the labio-nostril floor angle is approximately $105^{\circ}$ and acute. In cleft lip patients, the labio-nostril floor angle is obtuse due to the soft tissue defect of the nostril sill and the supporting bony framework. ${ }^{[7]}$

Buried flaps have been used in the lip. ${ }^{[8-11]}$ In order to shorten the lip, Kostianovsky ${ }^{[8]}$ augmented the upper and lower lip using buried de-epithelialized local flaps. In order to improve the senile lip, Guerriss ${ }^{[9,10]}$ deepithelialized a strip of skin on the vermilion border and buried the remaining dermal flap in the pocket, which was performed by undermining the skin of the superior third of the upper lip.

In the above studies, the buried flaps were used to augment the upper or lower vermilion. In our study we used a de-epithelialized flap for simultaneous nasal base narrowing and nostril sill augmentation.
In order to replace deficient nostril sills, Millard ${ }^{[11]}$ denuded the epithelium of the alar base flap (D-flap) and pulled it under the lateral tip of flap $\mathrm{C}$. In the present study, we used a medially based de-epithelialized flap that did not require a pull-out suture or cinching suture in the nasal septum.

In an embryological study, Green ${ }^{[12]}$ found that two relevant muscular systems exist: sphincteric fibers below the mucosal surface that are more prominent at the nasal sill, and a more superficial layer corresponding to the muscles of facial expression. He noted that the lateral external muscle fibers pass around the lower free border of the alar cartilage to insert into the sphincteric muscle.

Recently, Oh et al. ${ }^{[13]}$ observed that the nasalis muscle lies most superficially in the nostril sill, followed by the depressor septi nasi and the orbicularis oris underneath. In our observations, we did not find the nasalis muscle in the middle of the nostril sill. Instead, obliquely running depressor septi nasi muscle fibers comprised the nostril sill [Figure 3]. In this study, we observed a depressor septi nasi muscle that ran obliquely and comprised the nostril sill just beneath a thick layer of dermis.

The measurements we used in this patient cohort (alar distance, nostril floor width) supported the proposal that narrowing the alar base is beneficial. The RR of the nostril floor was $7.72 \% \pm 3.62 \%$. The RRs of the alar 
distance and alar curvature distance were $7.09 \% \pm$ $3.72 \%$ and $6.46 \% \pm 6.24 \%$, respectively [Table 1]. Nostril length increased on the non-cleft side $(6.17 \% \pm 15.60 \%$; $P=0.04)$. Although it was not a statistically significant finding $(P>0.05)$, nostril length decreased on the cleft side $(2.95 \% \pm 30.81 \%)$ [Tables 1 and 2]. This indicates that flap augmentation was beneficial.

We think that this is an advantageous technique that uses tissue that may be discarded to improve nasal symmetry. The medially based de-epithelialized flap may be a viable method for nasal base narrowing and nostril sill augmentation in cleft lip nasal deformities.

\section{Financial support and sponsorship}

This study was supported by Inha University (INHAResearch Grant).

\section{Conflicts of interest}

There are no conflicts of interest.

\section{Patient consent}

Informed consent for medical photographs was obtained.

\section{Ethics approval}

All procedures performed in studies involving human participants were in accordance with the ethical standards of the institutional and/or national research committee and with the 1964 Helsinki declaration and its later amendments or comparable ethical standards.

\section{REFERENCES}

1. Hamilton GS 3rd. Y-V alar base reduction. Ear Nose Throat J 2014;93:98-102.

2. Ismail AS. Nasal base narrowing: the alar flap advancement technique. Otolaryngol Head Neck Surg 2011;144:48-52.

3. Foda HM. Alar base reduction: the boomerang-shaped excision. Facial Plast Surg 2011;27:225-33.

4. Farkas LG, Kolar JC, Munro IR. Geography of the nose: a morphometric study. Aesthetic Plast Surg 1986;10:191-223.

5. Stewart A, Edler RJ. Efficacy and stability of the alar base cinch suture. Br J Oral Maxillofac Surg 2011;49:623-6.

6. Gruber RP, Freeman MB, Hsu C, Elyassnia D, Reddy V. Nasal base reduction by alar release: a laboratory evaluation. Plast Reconstr Surg 2009;123:709-15.

7. Uhm KI, Shin KS, Lee YH, Lew JD. Nostril sill augmentation in secondary cleft lip. Ann Plast Surg 1987;19:391-9.

8. Kostianovsky AS. Upper and lower lip augmentation by buried, deepithelialized local flaps: an alternative to the use of foreign material implants when shortening the lips. Aesthetic Plast Surg 1996;20:433-7.

9. Guerrissi JO, Sanchez LI. An approach to the senile upper lip. Plast Reconstr Surg 1993;92:1187-91.

10. Guerrissi JO. Surgical treatment of the senile upper lip. Plast Reconstr Surg 2000;106:938-40.

11. Millard DR. Cleft Craft. The Evolution of Its Surgery. In: The Unilateral Deformity (Vol 1). 1st ed. Philadelphia: Lippincott Williams \& Wilkins; 1976.

12. Green MF. The embryological, developmental and functional importance in the repair of the nasal musculature to reduce the deformity of the cleft lip nose. Scand J Plast Reconstr Surg Hand Surg 1987;21:1-5.

13. Oh M, Lee DC, Choi TH, Kim S. Anatomic study of the nostril sill: classification and histologic findings. Ann Plast Surg 2010;65:56-9. 